\title{
Two dimensional power spectral density measurements of X-ray optics with the Micromap interferometric microscope
}

\author{
Valeriy V. Yashchuk*, Andrew D. Franck, Steve C. Irick, Malcolm R. Howells, \\ Alastair A. MacDowell, Wayne R. McKinney \\ Lawrence Berkeley National Laboratory, 1 Cyclotron Road, Berkeley, CA, USA 94720
}

\begin{abstract}
A procedure and software have been developed to transform the area distribution of the residual surface heights available from the measurement with the Micromap interferometric microscope into a two-dimensional (2D) power spectral density (PSD) distribution of the surface height. The procedure incorporates correction of one of the spectral distortions of the PSD measurement. The distortion appears as a shape difference between the tangential and sagittal PSD spectra deduced from the 2D PSD distribution for an isotropic surface. A detailed investigation of the origin of the anisotropy was performed, and a mathematical model was developed and used to correct the distortion. The correction employs a modulation transfer function (MTF) of the detector deduced analytically based on an experimentally confirmed assumption about the origin of the anisotropy due to the asymmetry of the read-out process of the instrument's CCD camera. The correction function has only one free parameter, the effective width of the gate-shaped apparatus function which is the same for both directions. The value of the parameter, equal to 1.35 pixels, was found while measuring the 2D PSD distribution of the instrument self-noise, independent of spatial frequency. The effectiveness of the developed procedure is demonstrated with a number of PSD measurements with different X-ray optics including mirrors and a grating.
\end{abstract}

Keywords: interferometric microscope, power spectral density, X-ray optics, optical metrology

\section{INTRODUCTION}

The interferometric microscope, such as the Micromap- $570^{1}$, has become a basic metrology tool for highly accurate testing of the surface finish of X-ray optics with sub-Angstrom rms roughness. The standard list of output parameters of an interferometric microscope measurement includes values of roughness averaged over an area and along a sample line. However the task of designing high performance low scatter X-ray optical systems ${ }^{2}$ requires the development of sophisticated X-ray scattering calculations based on rigorous information about the optics. One of the most insightful approaches to these calculations is based on the two-dimensional (2D) power spectral density (PSD) distribution of the surface height, ${ }^{3}$ allowing for the evaluation of three-dimensional distributions of X-rays scattered by the optics. ${ }^{4}$

The 2D PSD function $S_{2}$ may be viewed as a Fourier decomposition of the 2D surface height distribution $h(x, y)$ into harmonic basis functions: ${ }^{5}$

$$
S_{2}\left(s_{x}, s_{y}\right)=\lim _{A \rightarrow \infty}\left\langle\frac{1}{A}\left|\int_{-L_{y} / 2}^{L_{y} / 2} d y \int_{-L_{x} / 2}^{L_{x} / 2} h(x, y) e^{-2 \pi i(u x+v y)} d x\right|^{2}\right\rangle,
$$

where $L_{x}$ and $L_{y}$ are the tangential and sagittal dimensions of the measured surface region, $A=L_{x} L_{y} ; u$ and $v$ are the spatial frequency variables corresponding to the tangential, $x$, and sagittal, $y$, coordinates. In the case of discreet measurements with pixel dimensions $\Delta x$ and $\Delta y, \mathrm{M}$ and $\mathrm{N}$ pixels in the tangential and sagittal directions, respectively, the 2D PSD distribution can be evaluated from the height distribution $h_{m, n}$ via equation

$$
S_{2}(l, k)=M N \Delta x \Delta y\left|F_{l, k}\right|^{2},
$$


where $F_{l, k}$ are the elements of the Fourier transform matrix,

$$
F_{l, k}=\frac{1}{M} \sum_{m=0}^{M-1}\left[\exp \left(\frac{-2 \pi i m l}{M}\right) \frac{1}{N} \sum_{n=0}^{N-1} h_{m, n} \exp \left(\frac{-2 \pi i n k}{N}\right)\right] .
$$

The corresponding estimates of the tangential and sagittal one-dimensional (1D) two-sided PSDs $S_{1}^{\prime}(l)$ and $S_{1}^{\prime}(k)$ can be obtained by summing over rows ( $l$ ) or columns ( $k$ ), respectively. Here $0 \leq l \leq M-1$ and $0 \leq k \leq N-1$, and prime signifies a two-sided PSD. These are then converted to one-sided (positive frequency only) just like the ones calculated from lines on the surface directly

$$
S_{1}(l)=2 S_{1}^{\prime}(l) g(l) \text { and } S_{1}(k)=2 S_{1}^{\prime}(k) g(k)
$$

where $0 \leq l \leq M / 2,0 \leq k \leq N / 2 ; g(l)=1 / 2$ at $l=0, M / 2, g(k)=1 / 2$ at $k=0, N / 2$, and $g(l)=1$ and $g(k)=1$ otherwise.

A straightforward attempt to transform the area distribution of the residual surface heights available from the interferometric microscope measurement into a 2D PSD distribution fails due to the spectral distortion in the PSD caused by an unknown spatial frequency response of the instrument. The response is characterized with the modulation transfer function (MTF), determining the bandwidth of the instrument. ${ }^{6}$

The overall MTF contains the contributions from the instrument optical system, detector, signal processing, software algorithm, and environmental factors. Generally, these contributions are difficult to account for separately. Nevertheless, in the present work we were able to isolate and to separately correct one of the sources of the PSD spectral distortion, appearing as a significant difference between the tangential and sagittal PSD spectra deduced from a 2D PSD measurement of an isotropic surface - Fig. 1. A detailed investigation of the origin of the anisotropy was performed that indicated the read-out asymmetry of the CCD camera and the necessary correction of the asymmetry by the software algorithm caused the problem. A similar asymmetry of the tangential and sagittal PSD spectra has also been observed with a phase measuring Fizeau-type interferometer. ${ }^{7}$

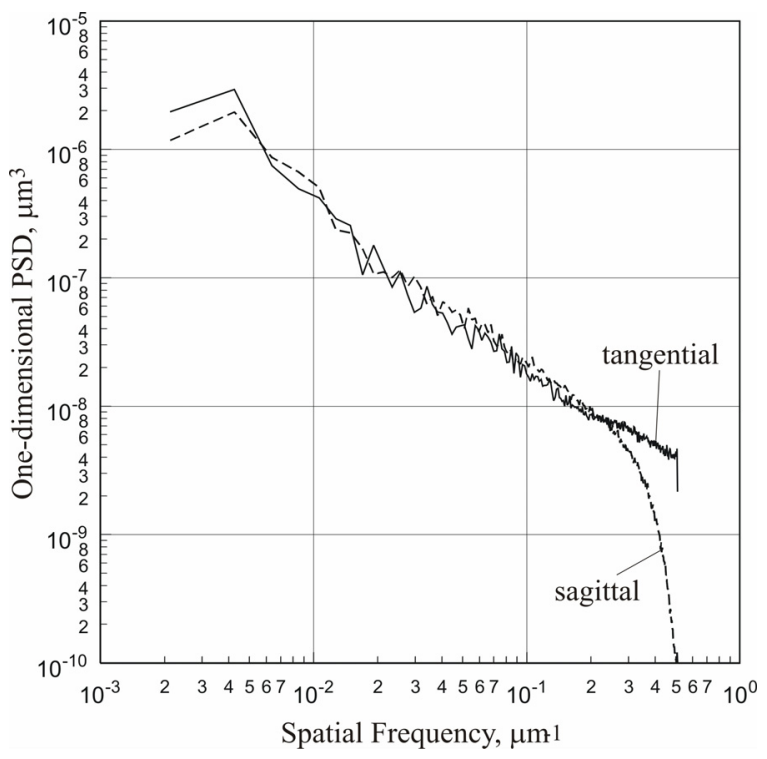

Figure 1: Solid line - tangential and dashed line sagittal PSD spectra of a super-polished crystal silicon mirror (rms roughness better than $1 \AA$ ). The spectra were extracted from the Micromap measurement with a $10 \times$ objective. The coordinate frame relates to the instrument: the tangential direction is along the horizontal axis, and the sagittal direction is along the vertical axis of the field of view of the CCD camera. Although the mirror had an isotropic surface finish, it was measured at orientation at $0^{\circ}, 45^{\circ}$, and $90^{\circ}$ with respect to the instrument frame in order to make sure that the resulting PSD spectra are the same for the different orientations. The asymmetrical distortion of the spectra appears as an abrupt roll-off at higher frequencies observed in the sagittal PSD but not seen in the tangential PSD.

In order to correct the asymmetry, a special procedure and additional software have been developed. The correction employs an analytically deduced detector MTF based on experimentally confirmed assumptions about the origin of the asymmetry. The correction function has only one free parameter, the width of the gate-shaped apparatus function which 
is assumed to be equal for both directions. In order to determine the value of the parameter, the developed procedure has been applied to the 2D PSD distribution for the Micromap-570 self-test measurement, which should give a white-noise 1D PSD spectrum independent of spatial frequency. The width parameter corresponding to the best fit white-noise spectrum was found to be 1.35 pixels.

The approach described in the present work can be used to correct PSD measurements from other interferometric microscopes, having similar read-out asymmetries. The effectiveness of the correction is demonstrated with a number of PSD measurements with different X-ray optics. In particular, the developed PSD procedure was successfully used to measure the spatial distribution of groove density of an X-ray grating.

The overall instrument MTF can be evaluated by comparing a PSD distribution of a known test surface with a corresponding ideal numerically simulated PSD. ${ }^{8,9}$ The square root of the ratio of the measured and simulated PSD distributions gives the MTF of the instrument. There is another, totally experimental, way to estimate the MTF, where the same surface is measured with different techniques with overlapping spatial frequency ranges. This involves the comparison of PSD measurements of X-ray mirrors performed with the Micromap interferometric microscope, and an atomic force microscope, and evaluated from mirror surface scattering and reflectivity measured with an X-ray scattering facility. This will be described elsewhere, ${ }^{10}$ and it is beyond the scope of this work.

Here we would like to emphasize that most of the practical methods used to determine the 2D MTF function of an interferometric microscope are based on the PSD measurement of a 1D test surface, such as a step height standard, ${ }^{8,9}$ or a surface with isotropic finish. ${ }^{10}$ In general without additional assumptions, it is impossible to recover the 2D MTF just from measured 1D PSD spectra. The assumption about symmetry (isotropy) of the instrumental spatial resolution allows one to bring the problem to searching for an effective 1D MTF. The technique developed in the present work helps to confirm the assumption about isotropy of the MTF.

\section{ORIGIN OF THE PSD SPECTRA ASYMMETRY}

A systematic investigation searching for the origin of the asymmetry between tangential and sagittal PSD spectra at higher spatial frequencies (Fig. 1) was performed. A test involving changing the mirror orientation with respect to the instrument demonstrated that the spectral distortion did not relate to the surface finish of the mirror. Moreover, with rotation of the CCD camera, we excluded all possible sources linked to the instrument elements before the CCD camera, namely the light source and the optics. Among the excluded possibilities, were the astigmatism of the objectives, and the rectangular shape of the lamp filament. ${ }^{11}$ Therefore, the distortion was caused either by the CCD camera itself or by the necessary correction of the asymmetry by the software algorithm that extracted the height distribution from the interference measurements.

In the course of the investigation, it was found that smoothing the measured height distribution over two tangential pixels washed out the asymmetry. Figure 2 shows the tangential and sagittal PSD spectra of a super-polished silicon mirror obtained after averaging the measured height distribution over two neighboring pixels in the tangential direction. The same measurement that is shown in Fig. 1 was processed. After averaging, the asymmetrical distortion of the PSD spectra disappeared, while the tangential PSD acquired the roll-off at the higher spatial frequencies, similar to the unprocessed sagittal PSD function.

The effect of symmetrizing with smoothing over two tangential pixels discovered empirically suggests that similar smoothing over two sagittal pixels was initially applied to the measured height distribution. Such a smoothing has already been incorporated into the Micromap software in order to suppress the distortion due to the CCD read-out process. ${ }^{12}$ This kind of problem appears when a two field interline camera is used. ${ }^{13}$ In our case, one field consists of odd pixel lines; another one includes the even lines. Both fields are collected simultaneously but are read out alternately. Such a read-out process leads to the systematic distortion between alternate detector outputs, corresponding to two fields. This distortion is reduced by averaging (summing) alternate outputs. The averaging eliminates the distortion but also reduces image resolution in the sagittal direction. As a result, the Nyquist frequency in the sagittal direction is decreased by a factor of two and a high frequency roll-off in the sagittal PSD spectrum is observed. 


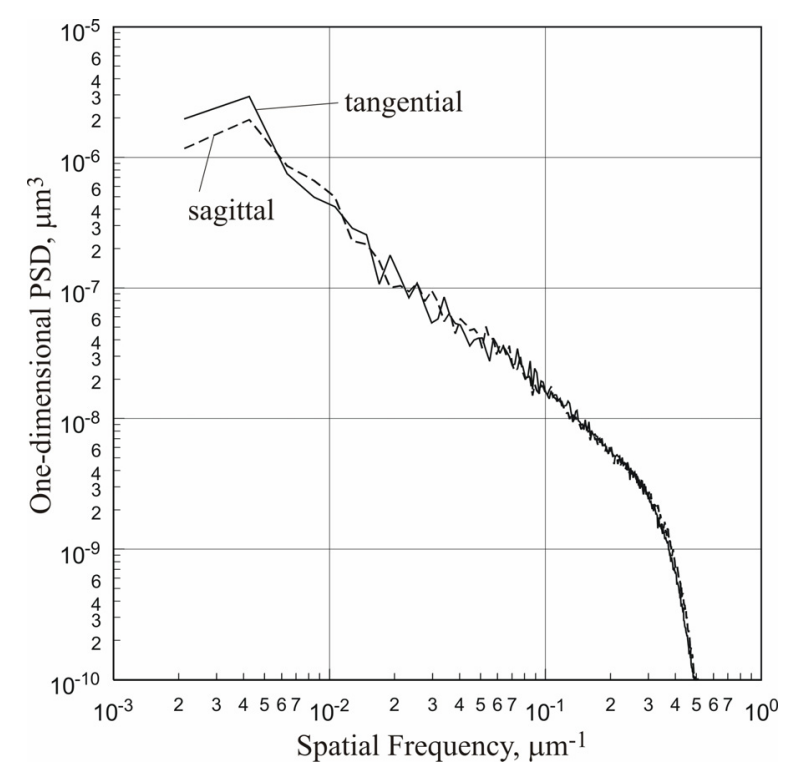

Figure 2: The tangential and sagittal PSD spectra of a super-polished crystal silicon mirror (rms roughness better than $1 \AA$ ) after averaging the measured height distribution over two neighboring pixels in the tangential direction. The same measurement as shown in Fig. 1 was processed. Solid line - tangential and dashed line - sagittal PSD spectra. The coordinate frame relates to the instrument: the tangential direction is along the horizontal axis, and the sagittal direction is along the vertical axis of the field of view of the CCD camera. The averaging washed out the asymmetrical distortion of the spectra, which had appeared as an abrupt roll-off at higher frequencies observed in the sagittal PSD but not seen in the tangential PSD Fig. 1.

The averaging over $\Delta p_{y}$ neighbor pixels in $y$-direction can be mathematically simulated with convolution of the untreated 2D height distribution inherent to the surface, $h(x, y)$, with a one-dimensional rectangular gate function, $\Pi(y)$, such as

$$
\Pi(y)=1 /\left(\Delta p_{\mathrm{y}} \Delta y\right) \text { at } y \in\left(-\Delta p_{\mathrm{y}} \Delta y / 2, \Delta p_{\mathrm{y}} \Delta y / 2\right) ; \text { and } \Pi(y)=0 \text { otherwise. }
$$

In the case of continuous measurement, the area height distribution convoluted along the sagittal direction, $y$, is given with an integral

$$
h^{y}(x, y)=\int_{-\infty}^{\infty} h(x, y-\lambda) \Pi(\lambda) d \lambda
$$

Fourier transformation $\mathrm{F}^{\mathrm{y}}(\mathrm{u}, \mathrm{v})$ of the distribution $h^{y}(x, y)$ can be expressed as a product of the Fourier transformation $\mathrm{F}(\mathrm{u}, \mathrm{v})$ of the unperturbed height function $h(x, y)$ and the Fourier transformation of the gate function (5) (see, e.g. Ref. 5):

$$
\mathrm{F}^{\mathrm{y}}(u, v)=\iint e^{-2 \pi i(u x+v y)}\left(\frac{1}{\Delta p_{\mathrm{y}} \Delta y} \int_{-\Delta p_{\mathrm{y}} \Delta y / 2}^{\Delta p_{\mathrm{y}} \Delta y / 2} h(x, y-\lambda) d \lambda\right) d x d \mathrm{y}=\mathrm{F}(u, v) \frac{1}{\Delta p_{\mathrm{y}} \Delta y} \int_{-\Delta p_{\mathrm{y}} \Delta y / 2}^{\Delta p_{\mathrm{y}} \Delta y / 2} e^{-2 \pi i v y} d y .
$$

The second factor in (7) is the detector modulation transfer function $\mathrm{A}_{\mathrm{d}}(\mathrm{u}, \mathrm{v})$ of a well-known analytical shape [see, e.g. Ref. 13, equation (8-2)]:

$$
\mathrm{A}_{\mathrm{d}}(u, v)=\left|\frac{1}{\Delta p_{\mathrm{y}} \Delta y} \int_{-\Delta p_{\mathrm{y}} \Delta y / 2}^{\Delta p_{\mathrm{y}} \Delta y / 2} e^{-2 \pi i v y} d y\right|=\left|\frac{\operatorname{Sin}\left(\pi \Delta p_{\mathrm{y}} \Delta y v\right)}{\pi \Delta p_{\mathrm{y}} \Delta y v}\right| .
$$

The function $\mathrm{A}_{\mathrm{d}}(u, v)$ has a first zero crossing when $\pi \Delta p_{\mathrm{y}} \Delta y v=\pi$ that corresponds to the detector spatial frequency cutoff at $v_{\mathrm{c}}=1 /\left(\Delta p_{\mathrm{y}} \Delta y\right)$. Convolving over two pixels $\left(\Delta p_{\mathrm{y}}=2\right)$ decreases the cutoff frequency by a factor of two. Therefore, the Nyquist frequency, which is the highest spatial frequency of the PSD function, is 
accordingly decreased by factor two to be equal to $v_{\mathrm{N}}(\Delta p=2)=1 /(4 \Delta y)$ instead of $v_{\mathrm{N}}=1 /(2 \Delta y)$ assumed in (4). Due to the convolution, the PSD information for higher frequencies is irretrievably lost. Our goal is to develop a procedure to correct the PSD distribution for the range below $v_{\mathrm{N}}(\Delta p)$ at a $\Delta p$, which has been experimentally optimized in order to get an isotropic overall MTF of the instrument, convenient for further correction. The procedure has to account for the discreet character of the microscope measurement and the way the averaging has been performed.

\section{DETECTOR MTF AT DISCREET MEASUREMENTS}

In the case of discreet measurement, the general expression for convolution over arbitrary $\Delta p_{\mathrm{y}}$ pixels will be ponderous. Nevertheless, averaging over two pixels can be described in a rather elegant manner. Here we point out the highlights of the derivation. For $\Delta p_{\mathrm{y}}=2$ pixels and discreet measurements, the convolution can be expressed as an arithmetic average over two neighbor pixels. Here we perform averaging along the tangential direction in order to simulate the averaging procedure used to symmetrize the 2D PSD distribution (see also Fig. 2):

$$
\begin{aligned}
& F_{l, k}^{y}=\frac{1}{M} \sum_{m=0}^{M-1}\left[\exp \left(\frac{-2 \pi i m l}{M}\right) \frac{1}{N} \sum_{n=0}^{N-1}\left\{h_{n, m}^{y} \exp \left(\frac{-2 \pi i n k}{N}\right)\right\}\right]= \\
& =\frac{1}{M} \sum_{m=0}^{M-1}\left[\exp \left(\frac{-2 \pi i m l}{M}\right) \frac{1}{N} \sum_{n=0}^{N-1}\left\{\frac{h_{m, n}+h_{m, n+1}}{2} \exp \left(\frac{-2 \pi i n k}{N}\right)\right\}\right] .
\end{aligned}
$$

After simple algebraic transformations, the last expression can be modified to

$$
F_{l, k}^{y}=\frac{F_{l, k}}{2}+\frac{F_{l, k}}{2} \exp \left(\frac{2 \pi i k}{N}\right)+\exp \left(\frac{2 \pi i k}{N}\right) \frac{1}{M} \sum_{m=0}^{M-1}\left[\exp \left(\frac{-2 \pi i m l}{M}\right) \frac{h_{m, N}-h_{m, 0}}{2 N}\right] .
$$

We need to make some assumptions regarding the value of $h_{m, N}$ because formally there is no such measurement at our choice of $n \in[0, N-1]$. Nevertheless, without losing generality we can self-contain the consideration by assuming $h_{m, N} \equiv h_{m, 0}$. In this case, the third term in the right-hand side of Eq. (9) vanishes and we get very the simple relation:

$$
F_{l, k}^{y}=\frac{F_{l, k}}{2}+\frac{F_{l, k}}{2} \exp \left(\frac{2 \pi i k}{N}\right)=\exp \left(\frac{\pi i k}{N}\right) \operatorname{Cos}\left(\frac{\pi k}{N}\right) F_{l, k} .
$$

The corresponding PSD distribution is [compare with (2)]

$$
S_{2}^{y}(l, k)=M N \Delta x \Delta y\left|F_{l, k}^{y}\right|^{2}=\operatorname{Cos}^{2}\left(\frac{\pi k}{N}\right)\left|F_{l, k}\right|^{2}=\operatorname{Cos}^{2}\left(\frac{\pi k}{N}\right) S_{2}(l, k) .
$$

Therefore, the detector modulation transfer function for averaging over two pixels is:

$$
B_{d}(l, k)=\left|\operatorname{Cos}\left(\frac{\pi k}{N}\right)\right|
$$


The detector function $B_{d}(l, k)$ has a cutoff at the spatial frequency corresponding to the Nyquist frequency of the detector, $u_{\mathrm{N}}=1 /(2 \Delta x)$.

\section{PROCEDURE FOR CORRECTION OF THE DETECTOR MTF}

The correction procedure implies two stages. First, the measured height distribution is averaged over two tangential pixels in order to symmetrize the resulting PSD distribution. At this stage, the measured 2D PSD distribution is effectively multiplied by the MTF function given by (12). The PSD distribution becomes symmetrical with the same characteristic high frequency roll-off in both directions - Fig. 2. Second, the isotropic correction procedure is applied. In order to account for a possible isotropic broadening (additional to the averaging and arising, for example, due to a leakage between pixels) of the detector spatial resolution, a detector MTF with a shape described by equation (8) was used.

The correction procedure can be analytically described with a correction function $C_{d}(l, k)$

$$
S_{2}^{C}(l, k)=\mathrm{A}_{\mathrm{d}}^{-2}(k / N \cdot 1 / \Delta x, l / M \cdot 1 / \Delta y) B_{d}^{2}(l, k) S_{2}^{M}(l, k)=C_{d}^{2}(l, k) S_{2}^{M}(l, k),
$$

where the indices ' $M$ ' and ' $C$ ' at the PSD functions denote the measured and the corrected PSD distributions, respectively; in order to get (13), we used the relations $u=k /(N \Delta x)$ and $v=l /(M \Delta y)$. Using expressions (8) and (12), $C_{d}(l, k)$ is given by formula

$$
C_{d}(l, k)=\left|\frac{1}{N M} \frac{\pi^{2} \Delta p^{2} l k}{\operatorname{Sin}(\pi \Delta p l / M) \operatorname{Sin}(\pi \Delta p k / N)} \operatorname{Cos}\left(\frac{\pi k}{N}\right)\right|
$$

with the only free parameter of the effective pixel width, $\Delta p$, equal for the tangential and sagittal directions. We should note here that the expressions (8) and (12) have different dependences on spatial frequency. This means that the performed discreet averaging is not equivalent to smoothing with the gate function with width of two and, therefore, the optimal value of $\Delta p$ need not be equal or even close to two. The free parameter in the correction function just allows us to optimize the correction.

In order to find an appropriate value of the parameter $\Delta p$, the correction procedure was applied to a random distribution of pixel signals measured with the microscope self-test mode. In this mode, two consequent measurements are performed for the same sample surface area. Then, the height distributions obtained are subtracted one from another, washing out all specific (systematic) information about finish of the sample surface and artifacts of the instrument optics. The resulting height distribution is due to the system noise, which is ideally random and independent of pixel position. The unperturbed (theoretical) PSD spectrum for the random distribution (white-noise spectrum) is seen as a constant line in the 1D PSD plot.

Figure 3 shows the 1D PSD spectra measured with the self-test mode and with $10 \times$ objective. Similar to Fig. 1, the difference between the sagittal and tangential spectra in Fig. $3 \mathrm{a}$ is due to the read-out asymmetry of the detector. The PSD spectra obtained after correction of the asymmetry with averaging over two pixels in the tangential direction is presented in Fig. 3b. Now the spectra are almost identical with the same characteristic roll-off at higher frequencies.

The correction of the measured 2D PSD distribution with different values of the correction parameter $\Delta p$ leads to the spectra shown in Fig. 4a. There is a strong dependence of the correction efficiency on $\Delta p$. At smaller values of the parameter, $\Delta p<1.3$ pixels, the spectra have significant roll-off in the vicinity of the decreased Nyquist frequency, 
which is approximately $0.12 \mu \mathrm{m}^{-1}$. The larger values, $\Delta p>1.4$ pixels, give rise to overcorrection of the detector MTF, seen as a roll-up at the higher spatial frequencies.
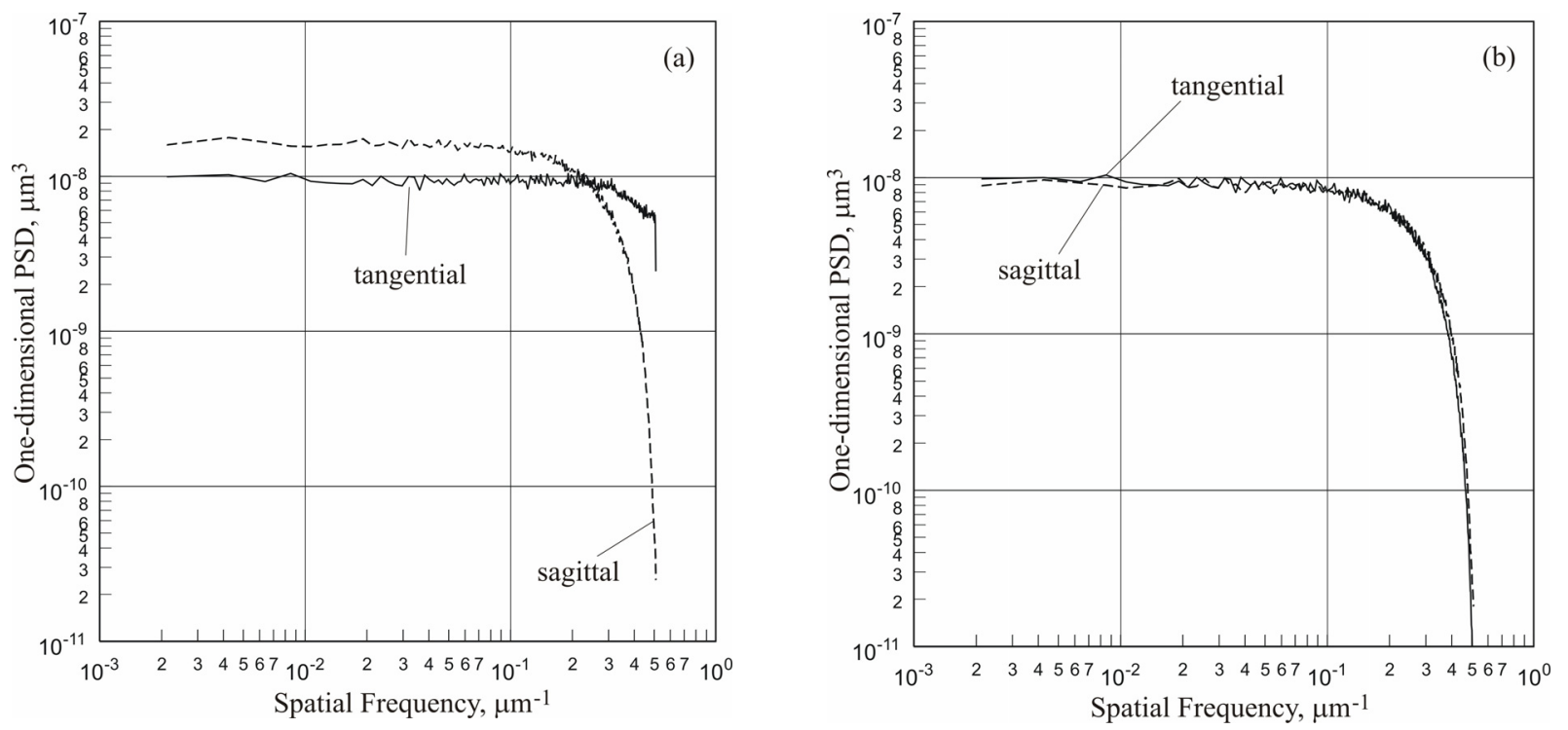

Figure 3: 1D PSD spectra measured with the self-test mode and with the $10 \times$ objective. a - There is no correction applied to the corresponding 2D PSD distribution. The difference between the sagittal and tangential spectra in plot (a) is due to the read-out asymmetry of the detector. It should be compared with Fig. 1a. b - The PSD spectra obtained after correction of the asymmetry with averaging over two pixels in tangential direction. The spectra are almost identical with characteristic roll-off at higher frequencies.
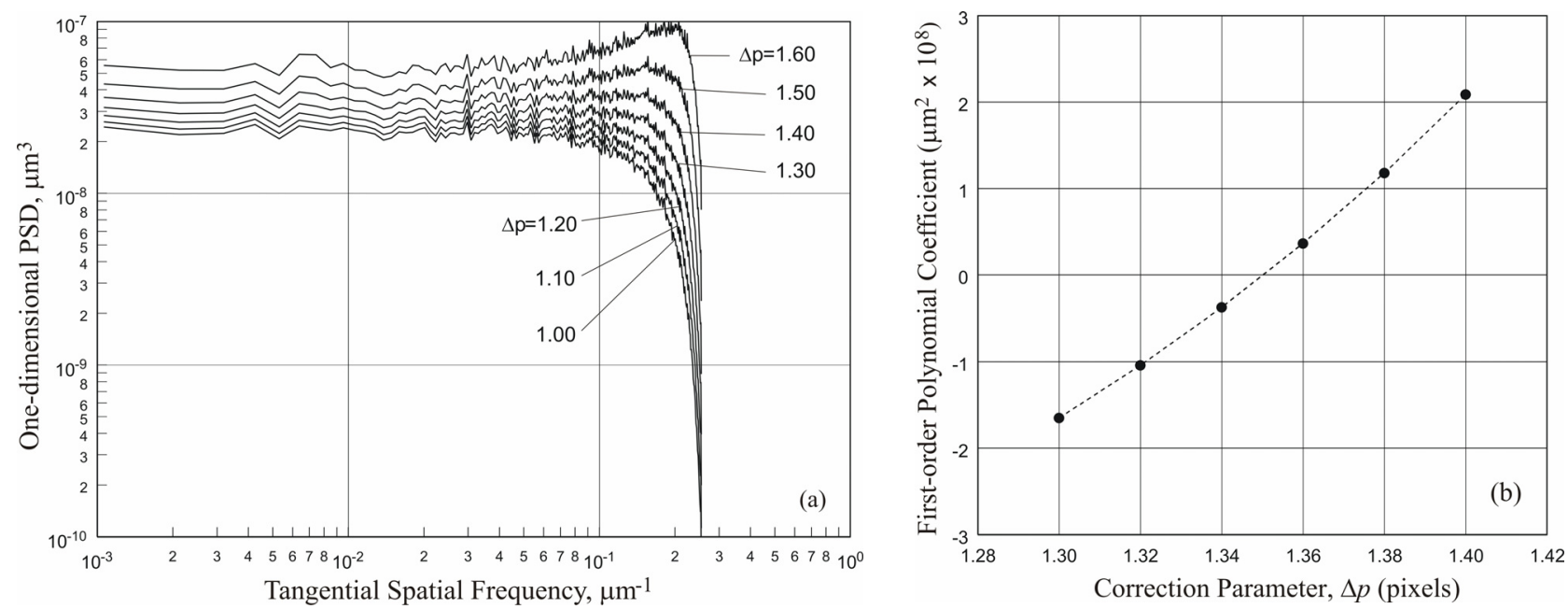

Figure 4: Dependence of the correction efficiency on the value of the parameter $\Delta p$. a - The tangential PSD spectra obtained from the 2D PSD distributions measured with the self-test mode and with $5 \times$ objective. The distributions were corrected with different values of $\Delta p$ from 1 pixel to 1.6 pixels. $\mathrm{b}$ - The dependence of the first-order polynomial coefficient while fitting the tangential PSD spectra over the spatial frequency range below $0.12 \mu \mathrm{m}^{-1}$. The optimal value of the correction parameter, corresponding to the zero-crossing in plot (b), is $\Delta p \approx 1.35$ pixels. The optimal value found is the same for all available objectives. 
The optimal value of the parameter $\Delta p$ was found when the corrected 1D PSD of the random noise was fitted to a first-order polynomial over the spatial frequency range limited with the decreased Nyquist frequency, $u_{\mathrm{N}}(\Delta p)$. Deviation of the resulting1D PSD spectra from the theoretical white-noise (a constant) dependence is described with the first-order polynomial coefficient. This is a measure of the efficiency of the correction procedure. Figure $4 \mathrm{~b}$ shows the dependence of the value of the first-order polynomial coefficient on $\Delta p$ used for the correction. The zero-crossing of the dependence corresponds to $\Delta p \approx 1.35$ pixel employed as an optimal value of the parameter. The optimal value is empirically found to be the same for all available objectives: $2.5 \times, 5 \times, 10 \times, 20 \times$, and $50 \times$.

Figure 5 illustrates the result of application of the correction procedure to the PSD measurement with the superpolished silicon mirror. In Fig. 5a, the PSD spectra are shown after the correction procedure when $\Delta p=1.35$ pixels was applied to the measured 2D PSD distributions. For Fig. 5c, the noise 2D PSD distribution measured at the self-test mode and corrected (Fig. 5b) was additionally subtracted from the corrected 2D PSD measurement presented in Fig. 5a. The correction washed out the asymmetrical distortion of the spectra (compare with Fig. 1). The roll-off in Fig. 5c is probably a manifestation of the overall instrumental MTF, which has isotropic shape. This is likely to be the dominated by Numerical Aperture effects of the objective lens which strongly affects the range of spatial frequencies sampled.
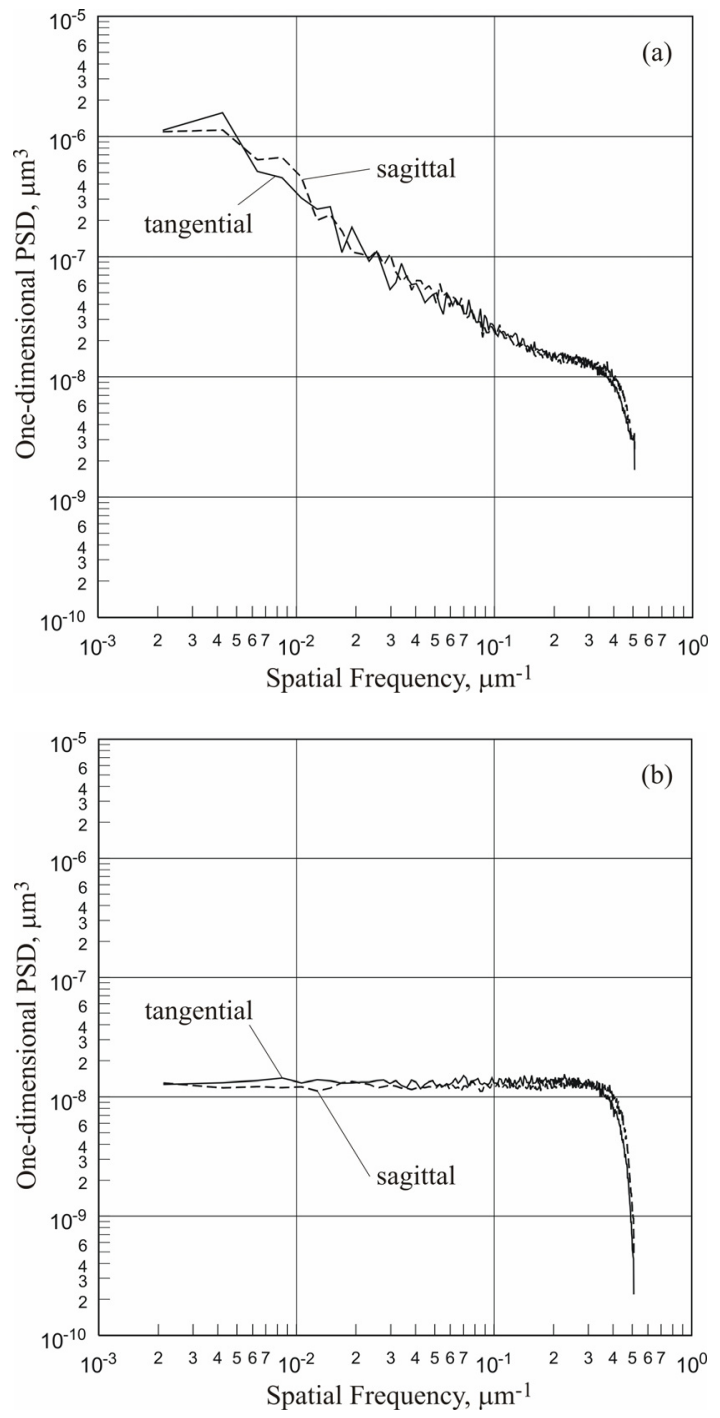

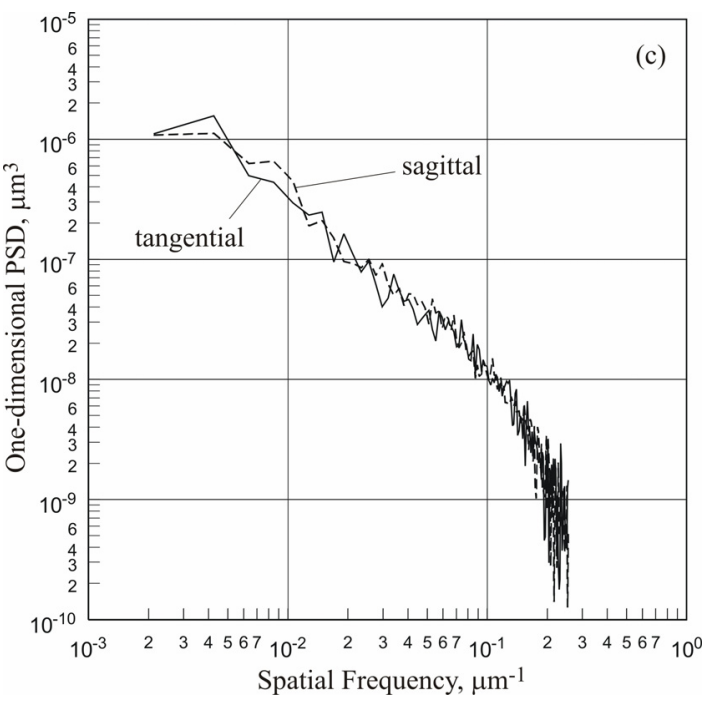

Figure 5: PSD measurements with the super-polished silicon mirror. a - The tangential and sagittal PSD spectra obtained from the 2D PSD distribution corrected with $\Delta p=1.35$ pixels. $\mathrm{b}$ - The PSD spectra of the instrument noise measured with the self-test mode and corrected with $\Delta p=1.35$ pixels. c - The PSD spectra of the surface finish obtained by subtracting the noise 2D PSD (b) from the measured 2D PSD distribution (a). The same measurement as shown in Fig. 1 and Fig. 2 was processed. Solid line the tangential and dashed line - the sagittal PSD spectra. The correction washed out the asymmetrical distortion of the spectra. The residual roll-off is a manifestation of the overall instrumental MTF, which probably has an isotropic shape. 
In the above example, the super-polished mirror possesses the surface roughness (rms) of approximately $1 \AA$. The instrument noise described has an effective roughness (rms) of about $0.6 \AA$ and contributes significantly into the measurement. Nevertheless, the PSD analysis based on this developed procedure allows one to effectively eliminate the noise contribution to the measured 2D PSD distribution and, therefore, to produce reliable information on the mirror surface finish.

\section{APPLICATION OF THE PSD ANALYSIS TO X-RAY OPTICS}

In this section we describe two examples of the application of the developed PSD procedure to metrology of X-ray Optics.

In the first example a cylindrically shaped mirror in the sagittal direction was under investigation. The surface curvature of the mirror is so large that the microscope measurement with low resolution objectives can not provide reliable data due to defocus at the sagittal edges of the field of view. However, the PSD measurement with all available objectives when analyzed all together allows one to obtain the faithful information about the surface finish.

Figure 6 presents the PSD spectra for the Micromap measurements of a platinum coated silicon mirror with surface rms roughness of approximately $3 \AA$. The $2 \mathrm{D}$ PSD distributions measured with all available objectives were corrected with the described procedure. The 2D PSDs of the instrument noise measured at the same experimental conditions with the self-test mode were also subtracted. The resulting tangential and sagittal PSD spectra are shown separately in Fig. 6a and Fig. 6b, respectively. The 2D PSD measurements with different objectives allow for extending the available spatial frequency range. A noticeable feature of such extension (Fig. 6a) is that the higher spatial frequency roll-off observed with an objective with lower resolution can be corrected with the measurement with a higher resolution objective. This can provide the possibility for additional correction of the PSD measurement for the overall MTF of the instrument, similar to the method discussed in Ref. ${ }^{10}$
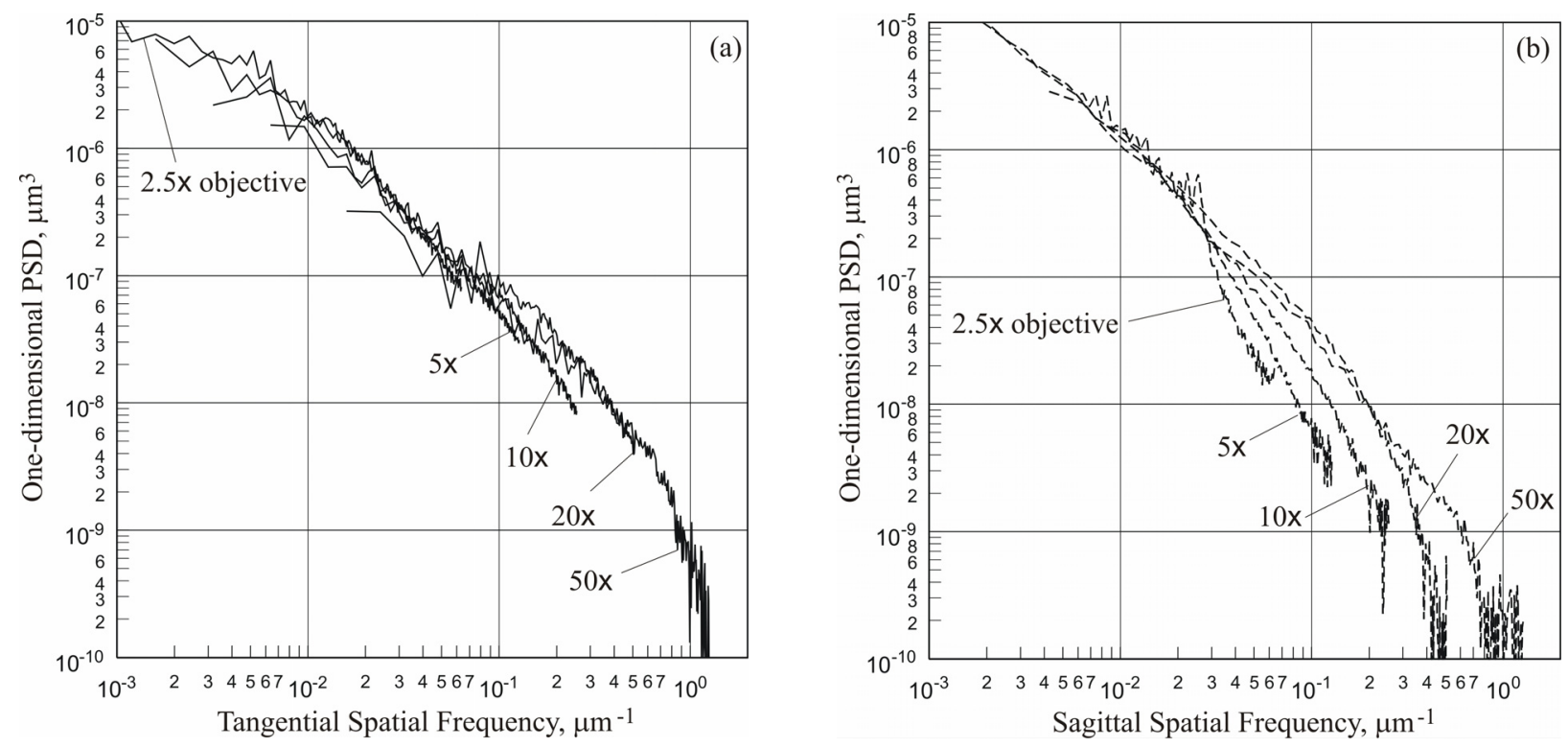

Figure 6: $\mathrm{a}$ - Tangential and $\mathrm{b}$ - sagittal 1D PSD spectra extracted from the Micromap measurement with different objectives. A silicon mirror with platinum coating with surface rms roughness of approximately $3 \AA$ was under investigation. The mirror was cylindrically shaped in sagittal direction with radius of curvature of approximately $24.9 \mathrm{~cm}$.

For the data shown in Fig. 6, the difference between sagittal (Fig. 6a) and tangential (Fig. 6b) spectra can be explained by the figure of the mirror. The mirror was cylindrically shaped in the sagittal direction with a radius of curvature of approximately $24.9 \mathrm{~cm}$. Such a mirror figure renders measurement difficult at low magnification because 
of the steep curvature in the sagittal direction reduces the field of view that is in focus. The Numerical Aperture is therefore restricted in the sagittal plane resulting in grater roll of to higher frequencies for the sagittal case (Fig. 6b) when compared to the tangential case (Fig. 6a). Despite this increased variability in the sagittal data the overall shape of both plots is similar and suggests a fairly isotropic surface. This suggests that the PSD measurements performed with different objectives are complementary, allowing for correction of the spatial frequency distortion due to the instrument imperfections and overall modulation transfer function of the lower resolution objectives via the PSD measurements with objectives with higher resolution.

The second example relates to the PSD analysis applied to the metrology of an X-ray grating with variable groove density. We have determined that PSD measurement provides very specific and rather rigorous information about the quality of X-ray gratings. It is shown, in particular, that the PSD measurement allows groove density characterization with enough accuracy to measure of the deviation of the density distribution from the desired linear law.

Figure 7 presents the PSD spectra obtained with a holographically recorded, ion beam etched, variable line spaced grating on a silicon substrate $\left(167 \times 36 \mathrm{~mm}^{2}\right)$ with a nickel coating. The grating (with area of $154 \times 10 \mathrm{~mm}^{2}$ ) was specified to have the groove density linearly distributed along the tangential direction. The actual groove density distribution shown in Fig. 8a was measured from the spatial frequency of the fundamental peak in the tangential PSD spectrum, which is the peak in Fig. 7 with the largest amplitude. The measured dependence was fitted with a second order polynomial in order to determine the deviation of the actual groove density from the desired linear shape - Fig. $8 \mathrm{~b}$. The observed parabolic-like deviation was characterized with a second-order polynomial coefficient measured to be $\beta=(0.055 \pm 0.006) \mu^{-1} \mathrm{~m}^{-2}$ (the position along the grating has dimension of meters). The standard deviation of the measured points around the best fit polynomial can be used to estimate the upper limit for the sensitivity of the PSD measurement to the groove distribution. For the $10 \times$ objective, the accuracy for the peak positioning was found to be

$$
\delta f \approx 10^{-4} \mu \mathrm{m}^{-1}=0.1 \mathrm{~mm}^{-1}
$$

This accuracy could be significantly improved with dedicated software which would account for the peak shape in the PSD spectrum specific for the grating.
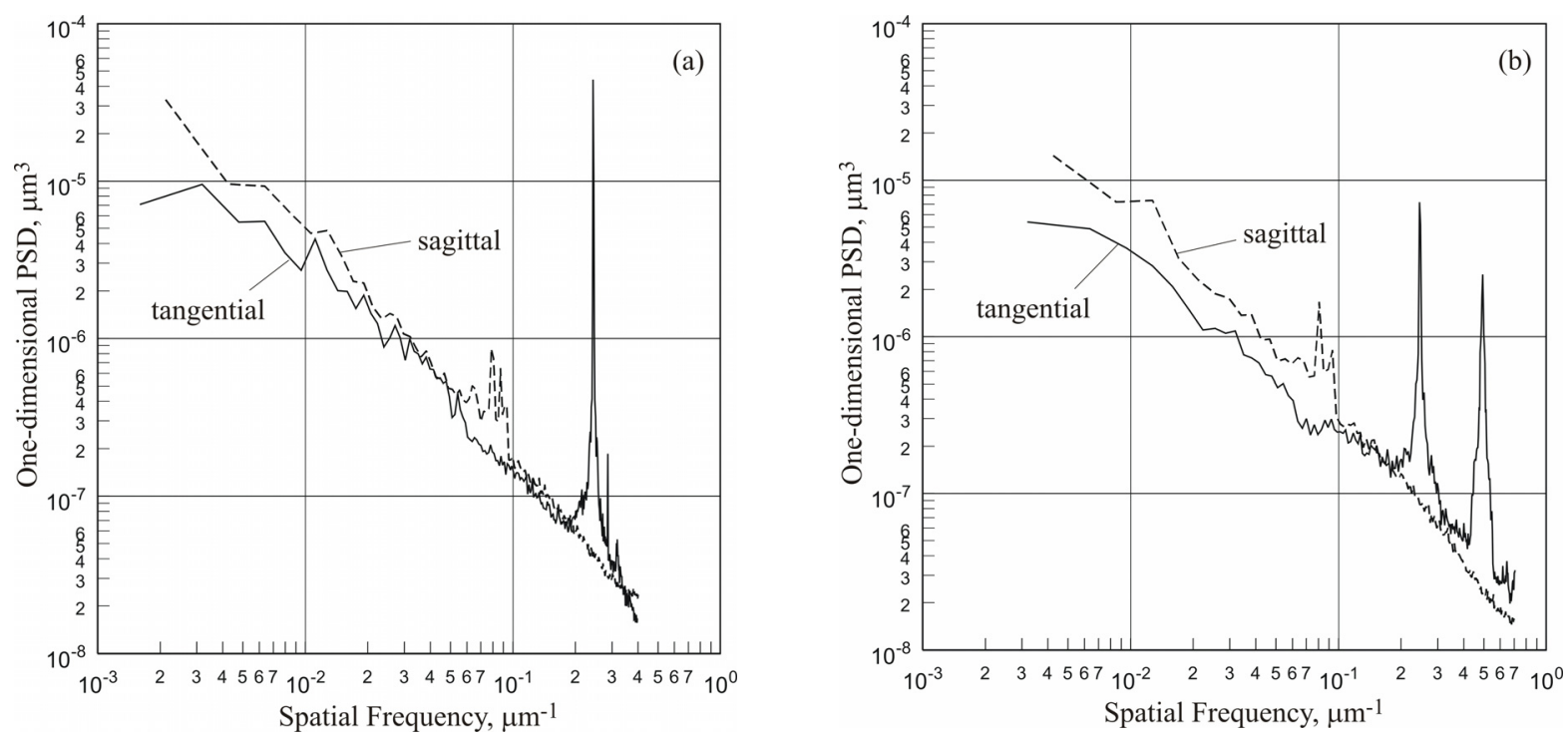

Figure 7: PSD spectra obtained with a holographically recorded, ion beam etched, variable line spaced grating on a silicon substrate $\left(167 \times 36 \mathrm{~mm}^{2}\right)$ with a nickel coating; $\mathrm{a}$ - the measurement with the $10 \times$ objective; $\mathrm{b}$ - the measurement with $20 \times$ objective. The spatial frequency corresponding to the largest peak is a measure for the groove density averaged over the area under investigation. 

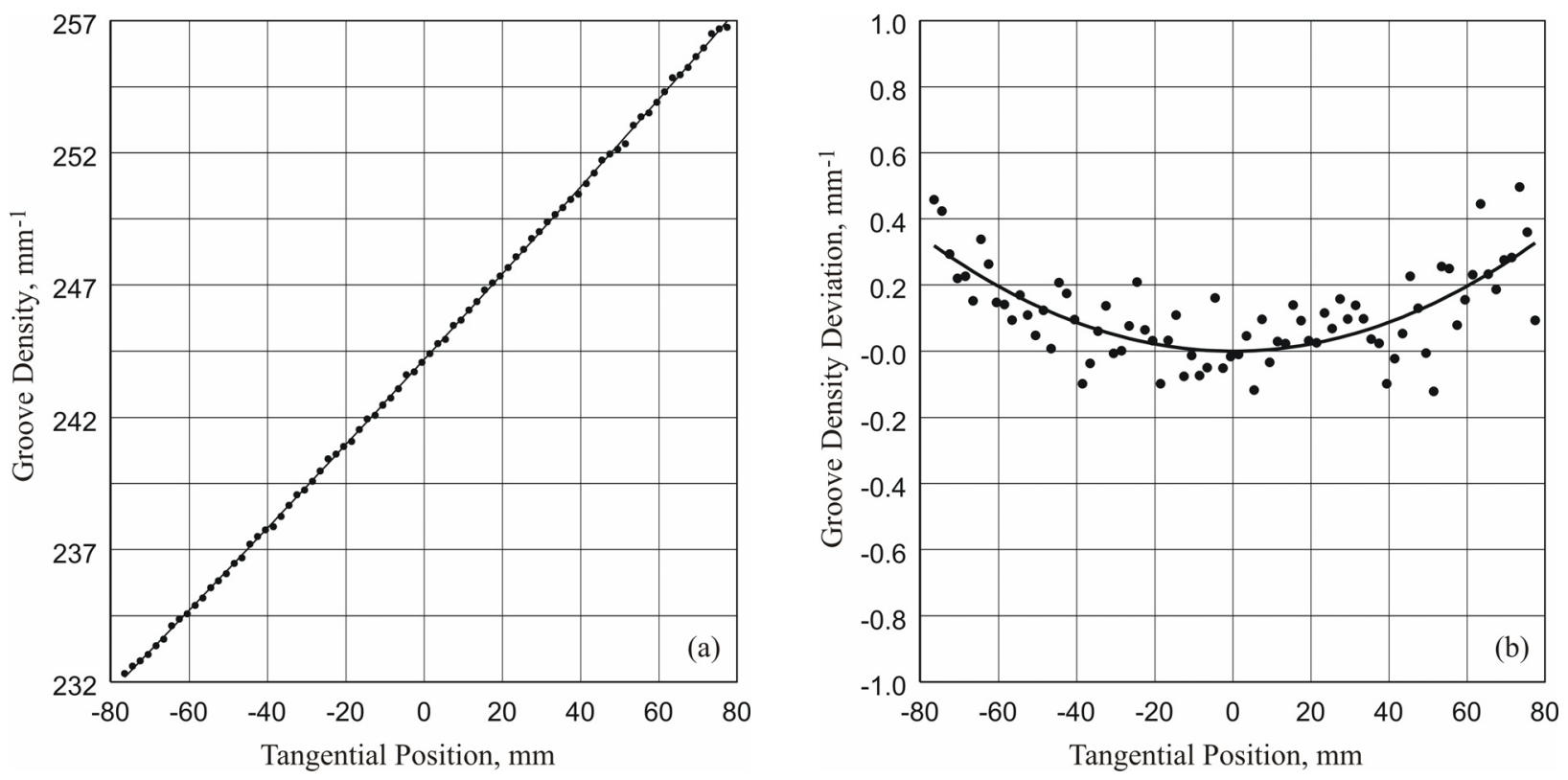

Figure 8: a - Tangential distribution of the groove density of an X-ray grating measured with the developed PSD procedure. $\mathrm{b}-$ Deviation of the groove density from a linear distribution. A grating, holographically recorded, ion beam etched with a silicon substrate $\left(167 \times 36 \mathrm{~mm}^{2}\right)$ and a nickel coating was under measurement with the Micromap interferometric microscope with the $10 \times$ objective. The best fit second order polynomial is shown with the solid line in plot (a). The solid line in plot (b) represents the second order term of the best fit.

We note that the amplitudes and widths of the fundamental and additional peaks at higher harmonics (Fig. 7) depend on the fine structure of the grating and could possibly also be used for metrology of the grating shape by comparing the measured PSD distribution with a desired pattern.

\section{DISCUSSION AND CONCLUSIONS}

In this work we have demonstrated that the data obtained with the MicroMap interferometric microscope designed for measuring surface roughness can be successfully converted into power spectral density distributions. Such a conversion necessarily involves a correction procedure. The correction procedure consists of two stages. First, the measured height distribution is averaged over two tangential pixels in order to symmetrize the resulting PSD distribution. This is required due to the asymmetry of the detector read-out process. Second, an isotropic correction procedure is applied based on an analytical correction function with the only one free parameter. The parameter was experimentally found from the white noise self-test PSD.

The efficiency of the correction procedure has been demonstrated with the PSD measurement of a number of X-ray mirrors and a grating, and with different experimental arrangements.

PSD measurements performed with different objectives have been shown to be complementary, allowing for correction of the spatial frequency distortion due to the overall modulation transfer function of the lower resolution objectives via the PSD measurements with objectives with higher resolution. Generally, the roughness measurement performed with an objective is compromised by its unknown MTF. According to our estimation based on the measurement with different objectives, the adjusting for the full instrumental MTF can increase the roughness value up to $20-30 \%$. Moreover, while the major contribution into the single number roughness value comes from the lower frequency part of the PSD spectra, ${ }^{14}$ the X-ray scattering depends mostly on the higher frequency part of the PSD spectra. These are the reasons for preferring PSD analysis of the quality of X-ray optics to more easily calculated surface roughness statistics. 
It has been shown that the PSD analysis is a powerful tool for the metrology of X-ray gratings. The PSD measurement of a grating with variable groove density has allowed for high precision characterization of the groove density with accuracy of $0.1 \mathrm{~mm}^{-1}$ and even better. This precision was enough to measure the parabolic deviation of the density distribution from the desired linear law of $\beta=(0.055 \pm 0.006) \mu^{-1} \mathrm{~m}^{-2}$.

In conclusion, the PSD measurement code with the correction procedure was developed as a program which runs on the public domain IDL Virtual Machine. ${ }^{15}$ It is available upon request to this metrology lab for use, development, and beta testing. Once our testing is fully completed, and corrections for the instrument MTF are included, it will be made available by web download for the use of the synchrotron optical and vendor communities via the ALS Optical Metrology Laboratory web-page. ${ }^{16}$ We fully expect that existing microroughness measuring instruments like the Micromap when combined with PSD analysis and X-ray scattering calculations will remain essential tools for the metrology of a new generation of X-ray optics.

\section{ACKNOWLEDGEMENTS}

The authors are grateful to Eric Gullikson, Philip Heimann, John Meiling, Howard Padmore, and Tony Warwick for extremely useful discussions. The Advanced Light Source is supported by the Director, Office of Science, Office of Basic Energy Sciences, Materials Science Division, of the U.S. Department of Energy under Contract No. DE-AC03-76SF00098 at Lawrence Berkeley National Laboratory.

\section{REFERENCES}

1. Micromap Corporation (Tucson, AZ; phone +1 520-881-1911; fax +1 520-881-1913).

2. L. Assoufid, O. Hignette, M. Howells, S. Irick, H. Lammert, P. Takacs, Future metrology needs for synchrotron radiation grazing-incidence optics, Nucl. Instrum. and Meth. in Phys. Research A 467-468 (2001) 267-70.

3. W. H. Press, S. A. Teukolsky, W. T. Vetterling, B. P. Flannery, Numerical Recipes in C++. The Art of Scientific Computing (Second edition; Cambridge University Press, New York, 2002).

4. D. Attwood, Soft X-rays and Extreme Ultraviolet Radiation (Cambridge University Press, New York, 1999).

5. R. N. Bracewell, The Fourier Transform and Its Applications (McGraw-Hill Publishing Company, New York, 1986), pp.108-112.

6. G. D. Boreman, Modulation Transfer Function in Optical and Electro-optical Systems (SPIE Press, 2001).

7. E. M. Gullikson (Lawrence Berkeley National Laboratory), private communication.

8. P. Z. Takacs, A step height standard for surface profile characterization, Proceedings of SPIE, 1993 (1993), 65-74.

9. C. R. Wolfe, J. D. Downie, and J. K. Lawson, Measuring the spatial frequency transfer function of phasemeasuring interferometers for laser optics, Proceedings of SPIE, 2870 (1996), 553-7.

10. V. V. Yashchuk, S. C. Irick, E. M. Gullikson, M. R. Howells, A. A. MacDowell, W. R. McKinney, F. Salmassi, and T. Warwick, Crosscheck of different techniques for two-dimensional power spectral density measurements of X-ray optics, will be published in Proceedings of SPIE - Advances in Metrology for X-ray and EUV Optics, part of the SPIE Optics and Photonics 2005 Symposium, San Diego, USA, July-August 2005.

11. The hypothesis about the lamp filament as a possible source of the asymmetry was suggested by J. Meiling, (Micromap Corp. ${ }^{1}$ ).

12. J. Meiling (Micromap Corp. ${ }^{1}$ ), private communication.

13. G. C. Holst, CCD arrays, Cameras, and Displays (SPIE Optical Engineering Press, Bellingham, 1998).

14. J. C. Stover, Optical Scattering: Measurement and Analysis (Second edition; SPIE Optical Engineering Press, Bellingham, 1995).

15. http://www.rsinc.com/idl.

16. http://www-esg.lbl.gov/Production/OML.

*vvyashchuk@lbl.gov; phone +1 510-495-2592; fax +1 510-486-7696. 